\title{
Analysis of Polarization Diversity at Terahertz Frequencies
}

\author{
Nicholas P. Lawrence*, Brian W.-H. Ng*, Hedley J. Hansen*†, Derek Abbott* \\ *School of Electrical and Electronic Engineering, \\ University of Adelaide, SA 5005, Australia. \\ nicholas.lawrence@adelaide.edu.au \\ ${ }^{\dagger}$ RFT Group EWRD, DSTO, \\ PO Box 1500, Edinburgh, \\ SA 5111, Australia.
}

\begin{abstract}
Terahertz frequencies offer the possibility of high resolution surveillance capabilities in areas requiring high levels of security. Terahertz imaging offers wavelengths providing high resolution with modest apertures while remaining able to penetrate clothing. In addition, they are not significantly affected by scatterers such as dust, fog and rain particles. Signal-to-noise ratio, dependent on antenna alignment, plays a critical role in providing high fidelity images. In this paper, performance of this ratio is analysed for the case of mutual orthogonality in three dimensions. Multiple-input multiple-output polarization diversity offers a way of improving system performance without the limitations associated with spatial diversity.

In the general case of a Rician fading channel the inclusion of a third orthogonal dipole may provide a useful additional degree of freedom and allow high capacity irrespective of antenna alignment.

We develop a novel Rician fading channel model for a tri-orthogonal half-wave dipole transmitter and receiver imaging system employing polarization diversity. Capacity is observed to be more robust than that of a dual-polarized system over the majority of a field of view. Simulation results have been compared to reference papers and show excellent agreement. The model design is applicable to other areas of high capacity systems research.
\end{abstract}

\section{INTRODUCTION}

Passive imaging techniques are readily scalable from millimeter-wave frequencies to terahertz frequencies. They are however prone to contrast issues due to high atmospheric attenuation at terahertz frequencies. Active imaging techniques, themselves reliant on target illumination by a terahertz source, are prone to misalignment issues. Multiple-input multiple-output (MIMO) signalling techniques exploiting spatial diversity through channel scattering have been widely adopted in wireless applications to increase performance [1] [2] [3]. Currently employed systems utilise uni-polarized or dual-polarized propagation due to their ease of implementation. However, these types of polarization do not fully account for a three dimensional environment. From a simple geometrical analysis, imaging performance is reliant on relative antenna position. This becomes an important limiting factor as design frequency increases as constant linear transmit power becomes typically harder to maintain; the consequence of which, in a highly secure area, could be serious. Every possible design advantage needs to be sought for the imaging system to be optimal. In the infra red region, imaging limitations are well documented. As a result, much interest has been given to the terahertz region where high data rate together with an unallocated portion of spectrum open the door to many possibilities [4]. Imaging at terahertz frequencies offers higher spatial resolution than at microwave frequencies, but is limited by available transmit power. To maintain this spatial resolution advantage, systems require perfect antenna alignment [5].

Innovative design is required to benefit from the advantages of terahertz frequencies while minimising the disadvantages. An omnidirectional dipole [6], deployed in a mutually orthogonal arrangement may increase both coverage and resolution without incurring a transmit power penalty. Coverage and resolution may be further enhanced by the propagation environment itself.

Polarization diversity offers the benefit of MIMO techniques for enhancing imagery in the instance of LOS propagation, which is not always the case for a spatially diverse system. The benefit of a rich scattering environment of a fading channel may further enhance this performance [2].

The presented link for imaging data transfer is developed in three dimensions according to geometry in Figure 1. For a mutually tri-orthogonal arrangement, nine sub-channel paths are generated. Nonfading power transfer along each sub-channel path is given by the Friis formula,

$$
\frac{P_{\mathrm{R}}}{P_{\mathrm{T}}}=G_{\mathrm{T}}\left(\phi_{\mathrm{T}}\right) G_{\mathrm{R}}\left(\phi_{\mathrm{R}}\right)\left(\frac{\lambda}{4 \pi s}\right)^{2} e_{\mathrm{pol}} L_{\mathrm{atmos}}
$$

where $\mathrm{R}$ refers to the receiver dipole, $\mathrm{T}$ refers to the transmitter dipole, $P$ is power, $G$ is dipole gain, 


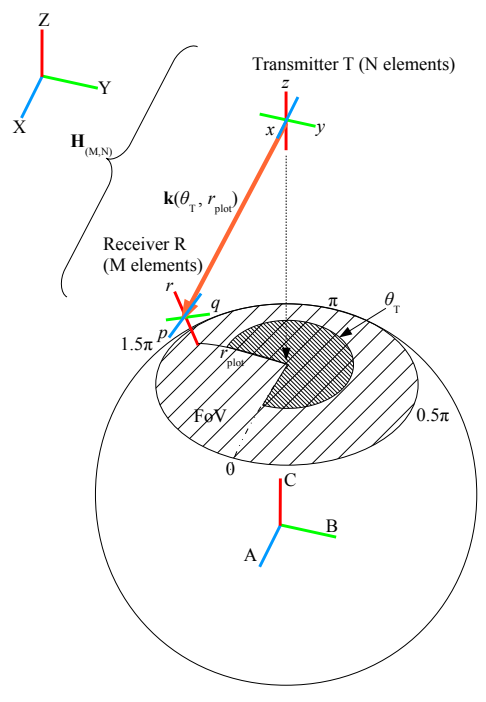

Fig. 1. Consideration of three dimensions in the model is achieved by a mutually orthogonal arrangement at both transmitter and receiver. The model achieves this through spherical geometry.

$\lambda$ is transmitted wavelength and $s$ is separation of transmitter and receiver. Polarization mismatch, $e_{\mathrm{pol}}$, is between a dipole at the transmitter and at the receiver. Atmospheric attenuation is given by $L_{\text {atmos }}$.

\section{Results}

Simulation is performed over the entire field of view (FoV) for the case of mutually orthogonal dipoles in three dimensions at transmitter and receiver; the former being fixed while the latter moves on a spherical surface to demonstrate both angular and spatial divergence. The case of non-fading channel performance is illustrated.

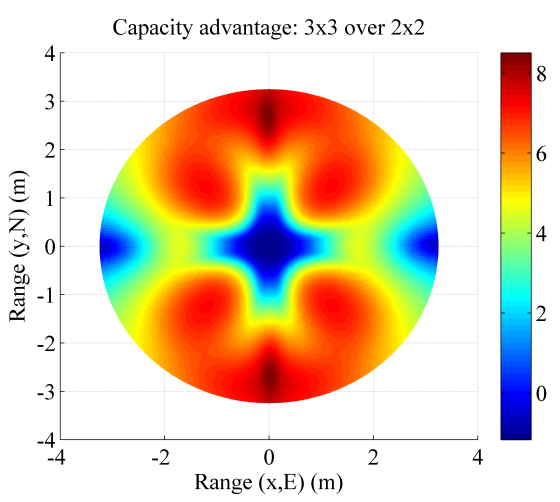

Fig. 2. Additive white Gaussian noise (AWGN) capacity advantage (bits/s/Hz): tri-orthogonality over dual-orthogonality (frequency = $122 \mathrm{GHz}$, bandwidth $=14 \mathrm{GHz}$, transmit power $=40 \mathrm{dBm}$, propagation length $=1 \mathrm{~m}$, sphere radius $=6 \mathrm{~m}$ )

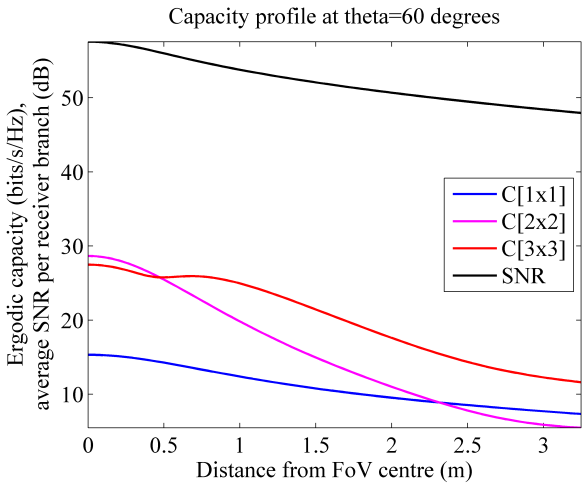

Fig. 3. AWGN channel capacity profile at $60^{\circ}$ or bisecting the positive $x, y$ quadrant (frequency $=122 \mathrm{GHz}$, bandwidth $=14 \mathrm{GHz}$, transmit power $=40 \mathrm{dBm}$, propagation length $=1 \mathrm{~m}$, sphere radius $=6 \mathrm{~m})$. Tri-orthogonal $(3 \times 3)$ system capacity remains high due to its additional degree of freedom or third dipole at transmitter and receiver.

\section{Summary}

Imaging performance is reliant on signal-to-noise ratio, itself on relative antenna positions. At terahertz frequencies, constant linear transmit power becomes typically harder to maintain; the consequence of which is either sub-optimal system performance or an exponential rise in system cost. Our simulations demonstrate excellent agreement at the field of view (FoV) centre with both simulated and measured capacity analysis given in line graph format in [7] and [8] which are readily applicable to terahertz frequencies. Tri-orthogonal capacity is observed as being more robust over the FoV, reducing misalignment issues. The model is appropriate to other types of application, including conventional communications systems.

\section{REFERENCES}

[1] W. Jakes, Microwave Mobile Communications. New York, NY: Wiley, 1974.

[2] A. Goldsmith, Wireless Communications. Cambridge, UK: Cambridge University Press, 2005.

[3] A. Paulraj, D. Gore, R. Nabar, and H. Bolcskei, "An overview of MIMO communications-a key to gigabit wireless," Proceedings of the IEEE, vol. 92, no. 2, pp. 198-218, 2004.

[4] J. Federici and L. Moeller, "Review of terahertz and subterahertz wireless communications," Journal of Applied Physics, vol. 107, art. no. 1111101, 2010, DOI: 10.1063/1.3386413.

[5] R. Macmillan, Advances in Sensing with Security Applications. New York, NY: Springer Verlag, edited by J. Byrnes, pp. 243268, 2006.

[6] M. Jacob, P. Herrero, and J. Schoebel, "Low-cost omnidirectional planar antennas for the $122 \mathrm{GHz}$ ISM frequency band," IEEE Antennas and Propagation Society International Symposium, 2008, DOI: 0.1109/APS.2008.4619478.

[7] J. Wang, H. Zhang, L. Tingting, and T. Gulliver, "Capacity of $60 \mathrm{GHz}$ wireless communication systems over fading channels," Journal of Networks, vol. 7, no.1, pp. 203-209, 2012.

[8] C.-Y. Chiu, J.-B. Yan, and R. Murch, "Compact three-port orthogonally polarized MIMO antennas," IEEE Antennas and Wireless Propagation Letters, vol. 6, pp. 619-622, 2007. 